\title{
Industrialización para la construcción de viviendas. Viviendas asequibles realizadas con prefabricados de hormigón
}

\author{
Industrialization for housing. Affordable precast concrete \\ housing \\ D. Fernández-Ordóñez ${ }^{(*)}$, J. Fernández Gómez ${ }^{(* *)}$
}

RESUMEN

Hay una necesidad creciente para la construcción de viviendas, necesidad que se ha incrementado de forma exponencial en las últimas décadas. Esta necesidad es considerable en los países en vías de desarrollo, donde la población tiene una tasa de crecimiento elevada, mucho mayor que en los países desarrollados. Además, la población en el Mundo está desplazándose progresivamente hacia las ciudades. Es en los alrededores de las ciudades de países en desarrollo donde surge una necesidad de construir viviendas de coste controlado, asequibles, que se convierte en algunos casos en crítica. La Comisión de Prefabricación de la fib (International Federation for Structural Concrete, Fédération Internationale du Béton, Federación Internacional del Hormigón Estructural) ha sido consciente por muchos años de este problema y tomó la decisión de crear un grupo de trabajo para desarrollar un documento sobre ${ }^{1^{*}}$ viviendas prefabricadas de coste asequible. Este documento estará dedicado a servir de ayuda sobre las técnicas de la prefabricación para las personas que deseen construir este tipo de viviendas. El presente artículo hace un resumen del trabajo desarrollado por este grupo hasta el momento.

195-16

Palabras clave: industrialización de viviendas, viviendas de coste controlado, viviendas asequibles, prefabricación, países en vías de desarrollo.

\author{
SUMMARY
}

The need for housing has increased significantly during the last decades. This need is considerable at present in third world and developing countries, where the population has a rate of growth much larger than developed countries. Furthermore the population in the world is shifting to the cities. In the surrounding areas of third world and developing countries is where the need for housing is sometimes critical. In these places there is a need for affordable, housing, for low income groups. The Commission of Prefabrication of fib has been conscious for many years to this problem which becomes vital when natural disasters put in to many of the housing that is at present been built in these areas. Therefore a Group was created to develop a document about Affordable Housing that could be a help to the people for the use of prefabrication techniques in the construction of houses. This article resumes the works that have been developed by this group up to this moment.

Keywords: industrialization of housing, low cost housing, affordable housing, prefabrication, developing countries.
(*) Vicepresidente de la Comisión de Prefabricación de la Fib. Universidad Politécnica de Madrid (Madrid, España)

(**) Miembro de la Comisión de Prefabricación de la Fib. Universidad Politécnica de Madrid (Madrid, España)

Persona de contacto/Corresponding author: david.fernandez-ordonez@upm.es (D. Fernández-Ordóñez)
Fecha de recepción: 07-02-09 Fecha de aceptación: 29-04-09 


\section{INTRODUCCIÓN}

Hay una necesidad creciente de construcción de viviendas, necesidad que se ha incrementado de forma exponencial en las últimas décadas. Es una necesidad considerable en los países en vías de desarrollo, donde la población tiene una tasa de crecimiento elevada, mucho mayor que en los países desarrollados (1).

Además la población en el Mundo está desplazándose progresivamente hacia las ciudades. Es en los alrededores de las ciudades de los países en desarrollo donde surge una necesidad urgente de construir viviendas de coste controlado, asequibles, que se convierte en algunos casos en crítica (2). Entendemos por viviendas asequibles aquéllas que pueden construirse o adquirirse por personas de un nivel económico comprendido entre el cuarto inferior y la mitad del poder adquisitivo medio en países en vías de desarrollo.

El reparto de la riqueza está cada vez más polarizado. Hay un gran aumento de capital humano, pero se sigue manteniendo una carencia de tecnología, conocimiento y formación para los proyectistas, constructores y otros miembros que intervienen en el proceso, normalmente especialistas alrededor del mundo de la construcción. El coste y la financiación del terreno donde construir es un problema clave para la construcción de viviendas en cualquier parte del mundo (3).

UNESCO establece que el derecho a la vivienda, el derecho al cobijo para la persona y la familia, es una condición de la ciudadanía. También "Human Rights Watch" establece que todas las personas tienen derecho a un nivel de vida adecuado y establece la vivienda dentro de este derecho.

En los países en vías de desarrollo hay una necesidad creciente de obtener cobijo, para protegerse de las condiciones naturales adversas. Sin embargo estos refugios de coste asequible deben cumplir, además, otras necesidades como es la protección contra el agua, aislamiento frente a condiciones térmicas adversas y la obtención de instalaciones sanitarias. Estas necesidades varían de forma apreciable entre unas partes del mundo y otras.

En los países desarrollados se considera la construcción de viviendas como un producto. En los países en vías de desarrollo la construcción de viviendas se convierte en un proceso en el que, en muchos casos, el propietario es también el constructor. El proceso de la autoconstrucción comienza con la obtención del terreno y puede durar entre diez y quince años. En la mayoría de los casos las personas viven en casas que no están finalizadas, sino que están en pleno proceso evolutivo continuo de construcción, de forma casi orgánica. En este proceso continuo de construcción hay una colaboración entre los distintos actores que participan en el proceso, como es la Administración, las organizaciones cooperativas y los constructores.

Con la industrialización y por tanto la prefabricación, entendida como una parte de la industrialización en la construcción de viviendas, se intenta construir viviendas de una forma organizada, que sigue un proceso. La intención puede ser construir gran cantidad de viviendas, de forma masiva, o también optimizar los procesos o incluso los materiales para la ejecución de la construcción.

Hay muchos ejemplos de sistemas industrializados en el mundo. De hecho hay también muchos que usan la prefabricación con elementos de hormigón. Gran parte de estos sistemas son desconocidos en otras partes del mundo, alejadas unas de otras, e incluso en lugares más cercanos no se transmite la información.

Tenemos que entender la industrialización de la construcción como el resultado de la aplicación de tecnología tanto a la producción (ingeniería de procesos) como al producto (ingeniería de producto).

Es de vital importancia tener en cuenta las posibilidades técnicas de la construcción en cada zona. Esto significa que la capacidad del equipamiento de producción, de transporte y de montaje son fundamentales para la definición de los elementos que se pueden utilizar en la construcción de viviendas. Por otro lado la capacidad de los medios y las infraestructuras puede ejercer de limitación para resolver qué solución es viable en cada área geográfica.

La economía de la solución posible cambia en cada zona porque en los países en vías de desarrollo los costes de los materiales de construcción y los procesos son distintos que en los países desarrollados. En muchas ocasiones los costes de materiales pueden ser incluso más altos en los países en vías de desarrollo que en los desarrollados. Por eso es necesario buscar tanto el tamaño de los elementos constructivos como la tecnología que sea adecuada para cada situación.

La industrialización es interesante en la medida que es un medio para armonizar la construcción, la industria y el resultado final que es la obra construida. Es atractivo poder aplicar las soluciones tecnológicas desarrolladas en países industrializados a las posibilida- 
des locales a los países en vías de desarrollo. Existen procesos constructivos y tecnología en la industria de los países desarrollados pero debe ser adaptada a las necesidades y capacidades de cada área geográfica. En muchos casos se pueden añadir elementos especiales, normalmente de desarrollo local, que complementan los sistemas industrializados originales. En ocasiones estos elementos locales son la clave para poder adaptar los sistemas más desarrollados.

Los elementos estructurales utilizados en cada sistema y cada zona deben normalmente asegurar un uso mínimo de los materiales, que, en general, tienen un coste que es limitativo en los países en vías de desarrollo. Este uso eficiente de los materiales debe ser combinado con una utilización estructural eficiente que asegure el uso posterior de la vivienda.

La edificación de viviendas puede ser considerada en su conjunto como una combinación de elementos funcionales. La viabilidad económica de la edificación en países en vías de desarrollo no es simplemente una cuestión de una construcción más económica o el uso de tecnologías más económicas. Lo que domina el coste total de la edificación de viviendas es normalmente más el precio de los materiales y el de los servicios añadidos a la construcción (4).

La metodología para la construcción de edificación asequible en países en vías de desarrollo tiene que ser menos sofisticada que en países desarrollados. Además debe implicar la necesidad de usar menos capital en la inversión.

Los cuatro retos fundamentales para resolver la carencia de viviendas asequibles en países en vías de desarrollo son: la falta de recursos, los fondos financieros insuficientes, la carencia de habilidades técnicas y la restricción de tiempo. La industria de la construcción necesita utilizar en mayor medida la industrialización y, en particular, prefabricación de hormigón para ejecutar proyectos de forma que pueda superar la carencia endémica de mano de obra especializada y pueda agilizar la construcción.

Hay varios niveles de complejidad en la industrialización de la construcción. Existen desde sistemas cerrados completamente prefabricados hasta sistemas parcialmente prefabricados que permiten el uso de materiales compuestos con los que se obtiene una gran libertad tanto para el diseño como para la construcción.

El hormigón es un material con grandes ventajas para ser utilizado en viviendas ase- quibles. La durabilidad es importante porque necesita poco mantenimiento, además tiene buena inercia térmica, puede ser utilizado para funciones estructurales y de cerramiento con buena terminación simultáneamente y no es sensible al ataque orgánico. También tiene algunas desventajas como mayores costes del cemento en países en vías de desarrollo comparado con países desarrollados o la posible escasez de sus componentes como son el propio cemento o los aditivos.

Hoy día reconocer la diversidad es una necesidad primaria de desarrollar procesos dentro de la industria de producir y montar elementos de construcción.

La Comisión de Prefabricación de la fib (International Federation for Structural Concrete, Fédération Internationale du Béton, Federación Internacional del Hormigón Estructural) ha sido consciente por muchos años de esta necesidad y tomó la decisión hace algunos años de crear un grupo de trabajo para desarrollar un documento sobre casas prefabricadas de coste asequible.

El grupo de trabajo realizó una labor de investigación, búsqueda y desarrollo de las soluciones disponibles en prefabricación de hormigón para ofrecer soluciones al problema. Los trabajos se han seleccionado por tipología, área geográfica, capacidad técnica y económica necesarias para su construcción. El trabajo se inició en 2004 y actualmente está en fase de conclusiones. El ámbito geográfico de estudio ha sido mundial, aunque ha habido más aportaciones de unas áreas que de otras.

Como resultado de este trabajo, está prácticamente concluido un documento que puede utilizarse como referencia de las técnicas de prefabricación utilizables para la solución de la carencia de viviendas en los países en vías de desarrollo, racionalizando los procesos de autoconstrucción y permitiendo su finalización en un plazo muy reducido.

\section{ASPECTOS GENERALES DE VIVIENDAS CONSTRUIDAS CON PREFABRICADOS DE HORMIGÓN DE COSTE ASEQUIBLE}

El derecho a la vivienda es un derecho de la persona y de la familia y es una condición de la ciudadanía. De todas formas los requisitos para un cobijo son distintos dependiendo de muchos y diversos aspectos, como es la situación geográfica, el clima, la sismicidad, la economía y los aspectos sociales.

La intención inicial es la de proyectar y construir viviendas que sean consecuentes con los usos y las rentas de los futuros usuarios. 
De acuerdo con este aspecto, son necesarios sistemas evolutivos que permitan hacer crecer la edificación desde modelos muy sencillos hasta otros más complejos.

La vivienda es una necesidad básica humana y por tanto estará en constante demanda en el futuro. Los requisitos humanos para el espacio varían ampliamente dependiendo de las condiciones climáticas y geográficas, así como de los aspectos socio económicos y culturales de la población.

Hay algunos principios que se deben tener en cuenta como es el proyectar un buen ambiente interior con suficiente luz y ventilación, la orientación, la protección frente al ruido, el polvo y los peligros locales. Se deben seguir al máximo las especificaciones locales.

En estos aspectos son esenciales la estandarización, la coordinación modular y la tipificación del diseño de los edificios. La racionalización de las dimensiones de los componentes y de la estructura terminada tiene influencia en la planificación de las viviendas. Con estas herramientas se puede conseguir la optimización del espacio, mejor productividad y eficiencia en la construcción de los edificios y en el uso de los materiales. (5).

La situación geográfica y el clima asociado son importantes para las necesidades de uso en este tipo de edificios. En zonas tropicales la necesidad de estanqueidad al agua se impone antes que otros requisitos. En estas áreas se debe prestar especial atención a las conexiones entre elementos estructurales y de cerramiento. Es también relevante la forma de expulsar el agua de la edificación y alrededor de ella, para evitar no sólo la entrada de humedades sino también daños a las cimentaciones.

Se necesitan algunos servicios esenciales para mantener unos mínimos estándares de salubridad. Éstos incluyen el agua corriente y los sistemas de evacuación de desperdicios o de aguas, tanto de elementos sanitarios como de lavado.

En este tipo de edificios no hay instalaciones de climatización y por esto es necesario tener muy en cuenta las condiciones de ventilación. Por ello es fundamental preparar la localización de las ventanas, en algunos casos sin cristales y simplemente cubiertas por celosías. En otros casos la ventilación se consigue con huecos en los techos para favorecer la ventilación natural. En otras circunstancias, la necesidad de pensar en una vivienda permanente hace imprescindible tener en cuenta los ataques orgánicos y otros peligros biológicos. En estas situaciones el uso del hormigón permite obtener estructuras resistentes frente a los deterioros naturales.

La acción del sismo puede ser un factor fundamental en la construcción de viviendas sociales o de coste controlado. Se han producido incontables desastres por una construcción no adecuada en áreas afectadas por terremotos. Es muy importante seguir las reglas establecidas respecto a la construcción en zonas sísmicas para que los edificios se comporten de forma adecuada en estas circunstancias tan especiales.

Algunos aspectos esenciales que hay que definir en caso de zona sísmica son los relativos al atado de las distintas partes de las cimentaciones y los muros. Otro aspecto es el atado de las partes estructurales de forma que un fallo local no lleve a una ruina absoluta de la construcción. Por otro lado, deben permitir que la estructura se deforme de forma controlada sin llegar a colapsar durante el sismo. Las reglas en cada caso dependen del tipo de estructura, en muchos casos la madera, fábrica, u hormigón ya sea prefabricado o in situ, acero, o, lo que es más común, una combinación de varios.

En muchos casos es fácil conseguir suficiente rigidez en edificios de baja altura sin tener que garantizar la ductilidad. Por otra parte puede ser muy interesante construir con materiales ligeros en áreas sísmicas porque, de esta forma, se reducen las fuerzas generadas.

El acceso a la zona de la construcción se convierte en relevante cuando se pueda decidir qué sistema utilizar en la construcción. En muchas ocasiones los medios de transporte y elevación definen la longitud y el peso posibles de los elementos. En otros, sólo es posible usar cierto tipo y tamaño de elementos que puedan ser movidos por dos personas.

La vivienda es una necesidad básica y, a la vez, la edificación de viviendas una parte vital del sector de la construcción, y, al mismo tiempo, es un factor importante de la economía. Como tal, la edificación de viviendas es un contribuyente principal en el empleo y la generación de rentas de las personas, ya que además les ayuda en su desarrollo socio económico. La economía es un aspecto que muchas veces es limitativo en una población para, simplemente, poder aspirar a cualquier tipo de vivienda. Por ello, en muchos casos para aspirar a una vivienda es necesario llegar a obtener suficientes fondos para comenzar la construcción, esta capacidad inicial limita el tamaño del proyecto que es posible acometer. (6)

Otro aspecto relevante es el acceso al terreno donde edificar. En muchas ocasiones la edi- 
ficación comienza de forma espontánea en terrenos libres, en forma de autoconstrucción, que luego se va asentando y haciendo formal. Se comienza al principio sin infraestructuras comunes como carreteras, abastecimiento de agua o saneamiento, por no hablar de instalaciones más avanzadas. Todas estas instalaciones comunes se desarrollan posteriormente a medida que lo hace la comunidad.

Hay algunos ejemplos de financiación adecuados para la construcción de viviendas de coste asumible. En unos casos se han desarrollado a partir de gobiernos locales, ayudas suficientes para poder comprar materiales de construcción que luego son utilizados en autoconstrucción. Algunas organizaciones no gubernamentales han llevado a cabo proyectos particulares con ayuda financiera y apoyo técnico en comunidades concretas con gran éxito. Las organizaciones financieras privadas normalmente no participan en proyectos de viviendas de coste asequible salvo en algunos casos recientes de organizaciones que han desarrollado sistemas de microcréditos con grán éxito.

Los aspectos sociales son relevantes cuando hay que decidir tanto la organización interna como la externa de las viviendas. En función de las convenciones sociales habrá, por ejemplo, habitaciones separadas, o una gran habitación con cocina aneja, o tener el baño fuera de la casa, como sucede en algunas comunidades de la India. Por tanto no hay que olvidar que hay que proyectar las viviendas de acuerdo con las necesidades y usos locales. Si se hace desde la lejanía es muy posible que las edificaciones puedan llegar a no ser utilizadas o sufran cambios drásticos por los usuarios.

Las viviendas asequibles deben cumplir con los mismos requerimientos sobre seguridad, uso, salubridad y ahorro energético que los edificios normales, simplemente variarán dependiendo de la zona donde se ubican.

\section{EJEMPLOS DE SISTEMAS PREFABRICADOS DE HORMIGÓN PARA VIVIENDAS ASEQUIBLES}

A continuación se exponen algunos de los criterios que deben seguirse para la construcción de viviendas con prefabricados de hormigón a precio asequible, proponiendo algunos ejemplos, tomados de los trabajos realizados en el seno del Grupo de Trabajo "Affordable Housing" de la Comisión de Prefabricación de la Fib. Los ejemplos se han seleccionado entre todos los casos estudiados como muestra de las diferentes alternativas posibles, seleccionando cuatro tipos compositivos y estructurales diferentes, dentro de los que nos parecen de mayor utilización $(6,7)$.

Hay otras referencias que se ampliarán en el trabajo que publicará la Fib. (9-11).

\subsection{Introducción}

Los requisitos siguientes son comunes a todas las edificaciones, pero en este caso de edificaciones de viviendas asequibles construidas con prefabricados de hormigón, deben ser considerados especialmente ya que se escapan de los controles normativos habituales. En este tipo de edificación hay además un alto grado de autoconstrucción y por ello creemos conveniente recordar los aspectos mas importantes.

Las cimentaciones deben ser construidas con materiales duraderos. Dependiendo de la localización, deben ser también resistentes a la acción del agua y sus posibles mecanismos de ataque. La resistencia a compresión de las cimentaciones necesariamente debe dar lugar a resistir la suma de las cargas de la estructura, incluyendo los pesos propios, cargas muertas y sobrecargas. Se pueden construir viviendas de coste asequible incluso sobre el terreno, sin forjado inferior. Sin embargo es recomendable construir un piso bajo para alejar a los habitantes de las humedades y variaciones de temperatura del suelo (12).

Las paredes de los edificios también deben tener suficiente resistencia a compresión para soportar las cargas verticales. Además deben proteger a los habitantes del viento y de la Iluvia y aislar en lo posible de la temperatura exterior. Si se usan estructuras reticuladas con vigas y pilares se pueden utilizar paneles para asumir, simplemente, los requisitos de cerramiento y aislamiento.

Los forjados de los edificios sobre el nivel del suelo deben soportar las cargas de los niveles superiores, si existen. Si su función es además el cerramiento del edificio deben ser estancos al agua para proteger a sus habitantes.

La prefabricación con hormigón se puede usar, en general, para toda clase de elementos estructurales en las viviendas. Las ventajas fundamentales del hormigón en la construcción de viviendas asequibles son: una muy buena resistencia a compresión, alta durabilidad y resistencia al fuego.

El hormigón, y especialmente el hormigón prefabricado, se puede usar para construir elementos lineales como vigas y pilares. También se usa de forma generalizada en cimentaciones o en elementos superficiales como son las paredes y los forjados. En muchos casos se usa el hormigón, in situ o prefabri- 
cado, en masa, pero para los elementos que tienen propiedades estructurales principales es necesario reforzarlos con armaduras de acero (13).

Se pueden usar bloques de hormigón para construir paredes, portantes o de separación. Se utiliza en estos casos un mortero de unión para los bloques. Solamente en casos excepcionales se añade armadura de refuerzo para muros de grandes dimensiones.

Puede suceder que para todas las soluciones de viviendas asequibles no sea necesario utilizar sistemas totalmente prefabricados $y_{\text {, }}$ en muchos casos, no es la mejor solución. El uso de sistemas mixtos con elementos locales y otros industrializados desarrollados en otros lugares, puede ser la mejor solución local en muchas ocasiones.

Las estructuras hechas parcial o totalmente con elementos prefabricados de hormigón tienen muchas ventajas, como son la resistencia, tanto para cargas estáticas como dinámicas, buena respuesta a fuertes vientos o a la acción de terremotos, durabilidad, resistencia al fuego, baja sensibilidad al ataque orgánico, buena inercia térmica y muchas otras.

Se debe tener en cuenta que la prefabricación basada en hormigón tiene muchas otras ventajas para la edificación de viviendas asequibles, como es la rapidez de montaje, sencillez de acuerdo con lo requerido y estanqueidad al agua. Normalmente se puede obtener una calidad adecuada sin tener que construir con sistemas de alta industrialización.

La construcción de viviendas asequibles está normalmente basada en sistemas sencillos.

Por ello los edificios con estructuras en planta simples, como pueden ser las rectangulares, se utilizan de forma frecuente y son ideales para la industrialización con elementos prefabricados de hormigón ya que exhiben una regularidad y repetición de elementos que hace más sencilla su producción y esto Ileva inevitablemente a soluciones más económicas (14).

Se pueden definir un gran número de soluciones técnicas y sistemas que pueden ser desarrollados con una industrialización de elementos de hormigón prefabricado. Sin embargo todos ellos se pueden agrupar en un número reducido de sistemas básicos para los que los principios básicos de diseño son similares. Nos referimos a un sistema estructural como una forma de conectar elementos estructurales verticales y horizontales, de forma que sean capaces de resistir las cargas verticales y horizontales normativas. Dependiendo del tipo de los elementos sustentantes que constituyen el sistema estructural se puede distinguir entre sistemas construidos con:

- elementos lineales, vigas y pilares

- elementos superficiales, paredes, paneles

- combinación de elementos lineales y superficiales

- sistemas espaciales, celdas monolíticas

Todos los sistemas principales tienen particularidades en los elementos utilizados como son distintos tipos de viga o forjados, paneles, etc. (15).

A continuación se muestran algunos ejemplos de sistemas prefabricados de hormigón utilizados para viviendas asequibles.

\subsection{Sistema de paneles integrales prefabricados en obra, de Uruguay}

El sistema se basa en elementos tipo viga prefabricados para la cimentación. Sobre ella se apoyan paneles portantes exteriores e interiores, armados con malla de acero y los exteriores, con un aligeramiento de poliextireno expandido de $2 \mathrm{~cm}$ de espesor.

Todos los paneles son de 12 centímetros de espesor e incluyen los huecos para ventanas y puertas. También incluyen los huecos para la posterior instalación de agua.

Se pueden utilizar forjados de distintos tipos, desde prelosas pretensadas prefabricadas hasta losas alveolares. Las juntas entre paneles y con las cimentaciones se rellenan de mortero durante el proceso de montaje. Las juntas entre paneles se refuerzan con algunos alambres de acero para dar continuidad estructural al conjunto.

Las dimensiones de los paneles son de 4 metros de longitud, 3,50 metros de ancho y 12 centímetros de espesor para no superar los $3.000 \mathrm{~kg}$ de peso (7).

En las Figuras 1 y 2 puede verse el esquema de la posición de los paneles y los detalles tipo de uniones del sistema.

\subsection{Sistema de paneles ligeros, de Sudáfrica}

El sistema comprende paneles prefabricados exteriores e interiores realizados con hormigón hecho con fibras de polipropileno. Los paneles son extremadamente delgados y por ello se ensanchan en los bordes. También se añaden pilares prefabricados de hormigón en las esquinas y en las uniones en T.

Los pilares, de sección cuadrada de 10 centímetros de lado, se montan en huecos preparados en la cimentación de 30 centímetros 
de profundidad, que luego se rellenan con grava. Los paneles prefabricados se colocan sobre cimentaciones lineales previstas para ellos. Los paneles se conectan con tornillos pasantes en los ensanches previstos. El suelo de la planta baja se hormigona en obra contra los paneles ya colocados.

Los paneles son de 2,70 metros de altura, 1,11 metros de ancho y 3 centímetros de espesor. Se adapta el ancho del panel para dejar los huecos de las puertas. Se definen de este espesor para que puedan ser montados a mano y suficientemente robustos para permitir seguridad de entrada de posibles ladrones.

Se puede montar una casa unifamiliar con seis trabajadores en tres días.

En las Figuras 3, 4 y 5 se presentan los detalles antes comentados.

\subsection{Sistema con elementos lineales, de Chile}

El sistema se basa en elementos prefabricados de cimentación, de estructura, del tipo pilar o viga, y luego forjados también prefabricados. Las paredes se pueden resolver con materiales variados en obra.

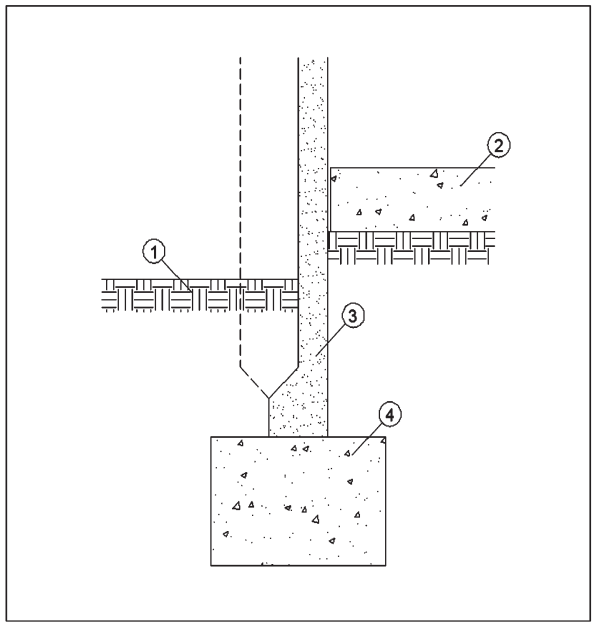

Los elementos prefabricados se ensamblan en obra y luego se unen estructuralmente mediante conexiones húmedas, con hormigón y barras de acero. No hay prevista instalaciones que se deben resolver en obra, pero sin necesidad de elementos especiales de elevación.

Las dimensiones utilizadas en los elementos son de 32 centímetros de espesor, 45 centímetros de ancho y 4,50 metros de longitud. Se construyen edificios de viviendas con un máximo de cinco alturas. (7)

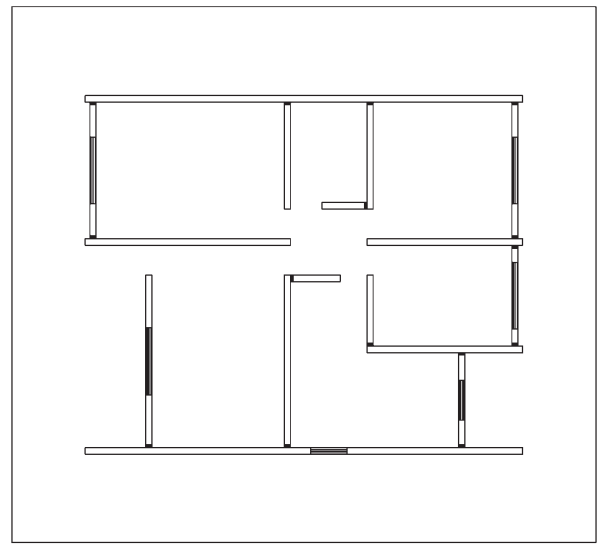

1. Sistema de paneles portantes. Esquemas. Uruguay. Disposición típica de paneles.

2. Sistema de paneles portantes. Esquemas, Uruguay A-Detalle de conexión lateral de borde, B-Detalle de conexión por dos caras, C-Detalle de conexión lateral, D-Detalle de conexión frontal, 1-Armadura de conexión, 2-Panel prefabricado.

3. Sistema de paneles ligeros. DetaIles. Sudáfrica. 1-Terreno, 2-Solera de hormigón, 3-Panel prefabricado de hormigón, 4-Cimentación in situ.

4. Sistema de paneles ligeros. Detalles. Sudáfrica. 1-Vigas secundarias de madera, 2-Cubrición,3-Vigas principales de madera, 4-Viga de conexión superior, de madera, 5-Panel prefabricado de hormigón, 6-Placa de cierre de fibrocemento.



(D) (A)

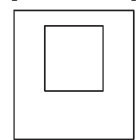

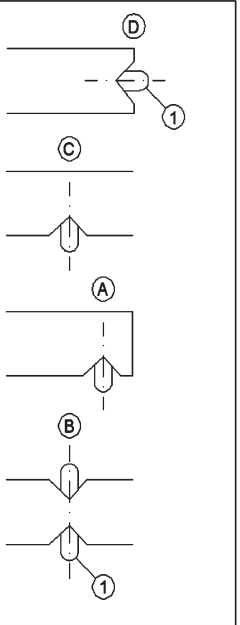

2



5. Sistema de paneles ligeros. Deta Iles. Sudáfrica. 1-Panel prefabricado de hormigón, 2-Soldadura puntual, 3-Marco de ventana Estándar, 4-Conectadores con tornillos. 
6. Sistema con elementos lineales. Esquema general. Chile. Pilar con armaduras salientes, vigas con ala superior para apoyo de losa de forjado y losa de forjado maciza.

7. Sistema con elementos lineales. Detalle del nudo de conexión húmedo con armaduras pasantes a través del hueco del pilar y la capa de compresión encima de las losas de forjado. Chile.

8. Sistema mixto de construcción. Sección. Costa Rica. 1- Viga de madera de coronación, 2-Panel prefabricado de hormigón, 3-Losa de hormigón "in situ", 4-Nivel de suelo terminado, 5-Cimentación in situ.

9. Sistema mixto de construcción. Detalle de conexión húmeda frontal. Costa Rica. 1- Mortero de relleno, 2-Panel prefabricado de hormigón.

10. Sistema mixto de construcción. Detalle de conexión húmeda de esquina. Costa Rica. 1- Mortero de relleno, 2-Armaduras salientes de los paneles, 3-Panel prefabricado de hormigón, 4-Hueco para la conexión.


8
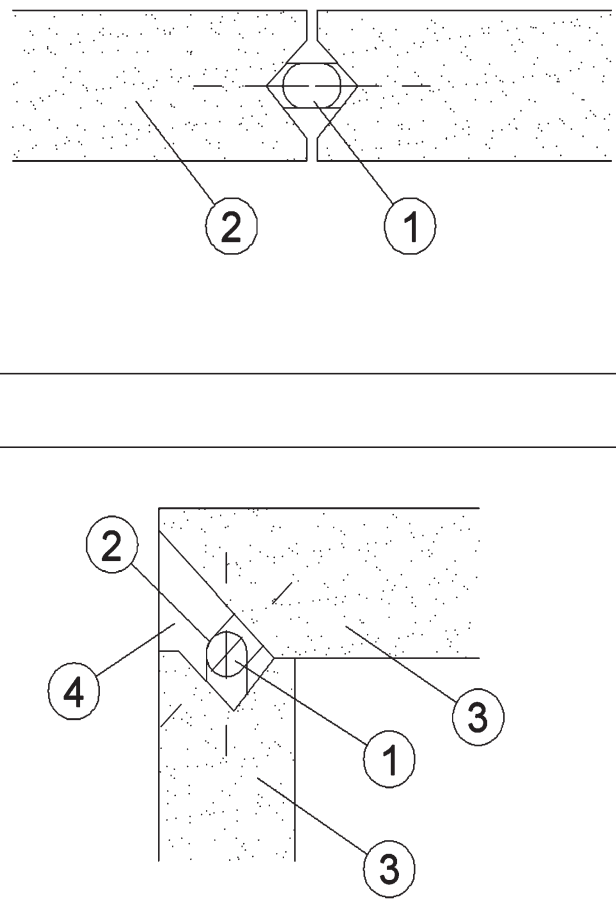

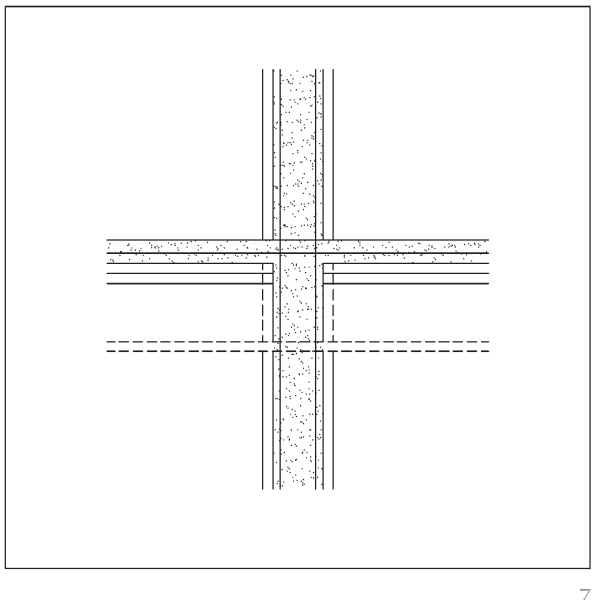

En las Figuras 6 y 7 se describe el esquema general y la unión entre los elementos.

\subsection{Sistema mixto de construcción, Zitro. Costa Rica}

El sistema se basa en paneles prefabricados para generar las paredes portantes y que se pueden colocar manualmente. Se introduce la parte inferior de los paneles en un hueco continuo tipo cáliz de las cimentaciones ya ejecutadas en obra. La parte superior del panel se sujeta a una viga de coronación. Esta viga de coronación se puede realizar con hormigón armado, metálica o de madera. De hecho la solución con viga de madera es la más utilizada.

Los paneles se construyen como módulos y pueden ser fácilmente adaptados para cualquier cambio arquitectónico. De la misma forma se pueden adaptar a distintas necesidades de huecos para puertas y ventanas (7).

En las Figuras 8, 9 y 10 puede verse gráficamente el sistema y las conexiones previstas.

\section{CONCLUSIÓN}

La creciente necesidad de alojar a las personas que masivamente se van desplazando del campo a las ciudades en los países emergentes provoca en la actualidad una urgencia para construir viviendas de costo asequible.

En la construcción de viviendas intervienen muchos actores hasta llegar a tener el resultado final. Dentro de los actores que pueden facilitar que realmente se puedan construir viviendas a costes asequibles está la industrialización de la construcción. Dentro de ésta, la prefabricación con hormigón presenta muchas ventajas sobre otras técnicas constructivas tradicionales.

Muchas experiencias, ideas y conceptos de la industrialización de la construcción de 
hormigón que se han generado en países más desarrollados pueden ser transferidas de forma sencilla a países en vías de desarrollo para que puedan ser utilizadas en la construcción de viviendas asequibles. Para transferir los conceptos de la industrialización a los países en vías de desarrollo hay que tener en cuenta sus particularidades locales. Cualquier intento de trasladar técnicas sin tener en cuenta aquéIlas está destinado al fracaso. La Comisión de Prefabricación de la fib decidió hace ya algunos años crear un grupo de trabajo que pudiera recoger la experiencia acumulada en varios países para luego trasladarla lo más ampliamente posible a otras zonas geográficas, Ilegando a generar un posible catálogo de ejemplos de sistemas prefabricados de hormigón adaptables según el caso. De esta forma, los técnicos y usuarios finales podrán tener una posibilidad entre otras soluciones para definir en primera persona y en cada lugar la mejor solución posible.

\section{BIBLIOGRAFÍA}

(1) BANCO MUNDIAL, 2005, World Development Indicators worldbank.org/ poverty/ wdrpoverty.

(2) COHRE, 2002, Centre on Housing Rights and Evictions, Forced Eviction Violations of Human Rights. Gobal Survey $n^{\circ} 8$, Ginebra Suiza.

(3) Salas, J.: La industrialización posible de la vivienda latinoamericana. Edit ESCALA, Bogotá, Colombia (2000).

(4) Salas, J., 1985, Respuestas a la Primera Consulta sobre la industria de los Materiales de Construcción de ONUDI, Mater. Construcc., Vol. 35, nº 198, pág. 15/ 30, Madrid, junio 1985.

(5) Salas J., 1993, Contra el Hambre de Vivienda. Soluciones tecnológicas latinoamericanas (Against hunger in housing. Technological solutions) Edit. Escala, Bogotá, Colombia.

(6) Salas J., Colavidas F., Oteiza I., 2007, Hacia una manualistica universal de componentes para el mejoramiento barrial.(Towards a universal components manual for better neighbourhods) Edit. Mairea, ETSAM - UPM, Madrid, 2007.

(7) CYTED: Catálogo Iberoamericano de Técnicas Constructivas Industrializadas para Vivienda de Interés Social. Proyecto Cyted XIV.2. (2001).

(8) Constructivas Industrializadas para Vivienda de Interés Social. (Industrial constructions for Social Housing) CYTED - Uruguay, Montevideo.

(9) Building for the people. COMDEV/MOZAMBIQUE.

(10) CVG Funvica, IDEC, CONCIT: Técnicas Constructivas Industrializadas para Viviendas de Bajo Costo en America Latina. Curso Teórico Práctico. Puerto Ordaz (Venezuela) 14 Oct/22 Nov 1991, (1991).

(11) Marcaccioli, L. and Menegotto, M. (2005), "Low-cost Houses for Self-construction with Precast Wall Panels". Role of Structructural Engineers towards Reduction of Poverty, IABSE, ISBN 3-85748-111-0, pp. $243-250$.

(12) Koehn, E. and Soni, M. (2001), Prefabricated housing in developing countries: India. Creative Systems in Structural and Construction Engineering, Edited by Singh, ISBN 905809161 9, pp. 57-61.

(13) fib, Planning and Design Handbook on Precast Building Structures, 2nd edition 2004.

(14) PCl, Design Handbook, Precast and Prestressed Concrete, 6th edition, 2004.

(15) Koncz, Handbuch Der Fertigteilbauweise, Bauverlag GmbH Wiesbaden und Berlin, 1967. 